\title{
Géophilosophie et déterritorialisation chez Gilles Deleuze : esquisse d'une nouvelle citoyenneté dans l'espace public postnational
}

\author{
Serge Bernard Emmanuel Aliana*
}

\section{Résumé}

En nette rupture avec la métaphysique traditionnelle qui pensait le sujet humain en terme d'un Moi hypostasié, stable, rationnel, identique à soi, conscient de soi, autonome et assigné à un lieu ou une « Polis » aux normes circonscrites, G. Deleuze, sous le prisme conceptuel de la « Géophilosophie » repense la condition destinale de l'homme d'une manière nouvelle. S’adossant sur les catégories nietzschéennes du tragique et du dionysiaque, Deleuze tente de défonder le sujet rationnel chéri par la philosophie classique pour soutenir l’idée d'un sujet irrationnel, esthétisant, déshistorisé, délocalisé, mobile, flexible, plurielle, décentré, nourrit à la sève d'une identité « rhizomique » et ouvert aux flux du monde. Dans la mesure où les flux sont toujours aléatoires et mutants et que le décentrement nie toute identité stable ou tout lieu d'assignation, Deleuze esquisse une véritable politique de l'hybridisme, du nomadisme, l'errance et de l'exil qui valorise à chaque fois les « lignes de fuites » comme principe de la « déterritorialisation ». Que signifie déterritorialiser sinon « faire passer des flux à l'état libre sur un corps sans organe désocialisé ». Arrachant le sujet humain des cadres institutionnels rigides et des pouvoirs étatiques établis, Deleuze entend émanciper celui-ci de tous les « codes » pour laisser s'exprimer en lui les désirs décodés. Il s’agit donc d’une libération du désir comme « processus schizophrénique ». Pour Deleuze, le devenir universel, c’est-à-dire l'Histoire a pour fin «la production désirante dans sa libération à l'égard de la production sociale ». C’est donc le désir qui est déterritorialisant.

* Département de Philosophie, Université de Yaoundé I, Cameroun. E-mail : alianaserge@yahoo.fr 


\begin{abstract}
In a clear break with traditional metaphysics, which conceived of the human subject in terms of a hypostatic Ego that is stable, rational, selfidentical, self-aware, autonomous and assigned to a place or a 'Polis' with circumscribed standards, G. Deleuze, through the conceptual prism of 'Geophilosophy' rethought the destinal condition of mankind in a new way. Drawing inspiration from the Nietzschean categories of the tragic and the Dionysian, Deleuze attempted to remove the foundation of the rational subject dear to classical philosophy and advanced the idea of a subject that is irrational, aestheticized, dehistoricized, delocalized, mobile, flexible, plural, decentred, fed by a 'rhizomatic' identity and open to the flows of the world. To the extent that these flows are always random and mutating and that decentring denies any stable identity or place of assignation. Deleuze outlined a real policy of hybridism, nomadism, wandering and exile that constantly highlighted 'lines of flight' as a principle of 'deterritorialization'. Indeed, what is deterritorialization if not 'causing flows to travel in a free state, on a desocialized body without organs'. By removing the human subject from rigid institutional frameworks and established state powers, Deleuze intends to emancipate it from all 'codes' to allow it to express its decoded desires. The focus is therefore on the liberation of desire as a 'schizophrenic process'. According to Deleuze, the universal future, i.e. history, is aimed at 'desiring production in its liberation from social production'. Thus, desire is a source of deterritorialization.
\end{abstract}

\title{
Introduction
}

Lorsqu'il publie dans les années 1960 L'espace public, avec, comme soustitre, « Archéologie de la publicité comme dimension constitutive de la société bourgeoise », Jürgen Habermas se fixe comme objectif essentiel de décrire l'idéal-type de «la sphère publique bourgeoise » à partir des contextes propres à des pays comme l'Allemagne, la France ou l'Angleterre du XVIIIe et du début du XIXe siècle. Cette description prend comme ancrage et circonstance historiques exceptionnels l'émergence du mode de production capitaliste. L'essor fulgurant du capitalisme, impulsé par le pouvoir économique grandissant de la bourgeoisie, impose une reconfiguration des rapports sociaux et une redéfinition de la notion de publicité.

Déstructurant l'ancien ordre féodal fondé sur les rapports hiérarchiques entre le roi et les vassaux, le capitalisme va faire apparaître un lien social nouveau qui prend comme matrice l'égalité et l'horizontalité des rapports entre sujets. L'éthique commerciale exige une égalité dans l'échange. En effet, quand on échange, il n’y a pas de verticalité ou de hiérarchie dans les termes. Les bourgeois ne sont soucieux que d'une chose : la bonne santé de 
leurs rapports d'échanges dans l'optique d'un enrichissement privé. Or quelque chose fait obstacle à cet enrichissement : le mercantilisme, c'est-àdire l'introduction de l'État dans les transactions et interactions privées. C'est ce mercantilisme qui oblige les bourgeois à se doter de presses, de journaux, de livres ou d'espace de discussion comme armes de contestation contre la présence trop envahissante de l’État. La bourgeoisie est animée par un motif fondamental : limiter l'autorité de l'État dans sa sphère d'activité. Il est question de « maintenir et redéfinir les frontières entre la société civile et l'État à travers deux processus interdépendants et simultanés : l'extension de l'égalité sociale et de la liberté et la restructuration de la démocratisation de l'État » (Habermas 1993:13). La bourgeoisie est dès lors amenée à se constituer en une opinion publique dont le rôle est de critiquer les abus étatiques et, par ce fait, former une force d'avant-garde au processus de démocratisation. C'est dans ce contexte particulier qu'Habermas va saisir l'espace public comme lieu d'expression et de confrontation d'opinions entre sujets égaux.

Le modèle théorique qui sous-tend l'argumentation d'Habermas est celui que formule Emmanuel Kant dans son ouvrage Qu'est-ce que les Lumières ? Dans cet ouvrage, Kant définit les Lumières comme « la sortie de l'homme hors de son état de minorité ». Dans le lexique kantien, la minorité est assimilée à l'hétéronomie, étymologiquement, le fait de recevoir sa loi ou sa détermination d'une autre personne. Concrètement, c'est le fait d'être sous la tutelle d'autrui. Prise dans ce sens, la minorité peut aussi vouloir dire l'état dans lequel les hommes sont plongés à l'époque de l'absolutisme. Les hommes sont assujettis au monarque, ce dernier se présentant lui-même comme issu du droit divin. ${ }^{1}$ La devise des Lumières, selon Kant, se résume en la formule suivante : Sapere aude ! Littéralement, ose savoir, c'est-à-dire aie le courage de penser par toi-même, aie le courage de faire un usage public de ta raison.

L'usage public de la raison, c'est, en d'autres termes, la confrontation des arguments qui, dans la pensée kantienne, est la pierre de touche de la vérité. Kant, il faut le préciser, se fait une idée précise de la vérité. Selon lui, elle n'est pas conçue par rapport à un objet, par rapport à un en soi des choses. Il n’y a de vérité que rapportée au sujet. La vérité a un ancrage subjectif, qui plus est, elle est d'essence intersubjective. C'est de la confrontation entre les individus que naît la vérité.

Dans ce moule conceptuel, Habermas va définir et circonscrire la notion d'espace public. L'espace public idéal, c'est le modèle idéal d'un public faisant un usage libre et partagé de sa raison à travers des plages de discussions, des organes et des tribunes d'expression. L'espace public devient 
dès lors un espace démocratique ouvert à tous, forum d'un discours d'assemblée permanent où doit s'exercer librement la raison critique des individus éclairés. Dans cette optique, l'espace public est solidaire de la construction d'un idéal républicain qui prend forme dans l'État-nation démocratique. On peut donc voir un lien génétique et même généalogique entre l'espace public, « sphère publique bourgeoise », et la mise en chantier de l'État-nation moderne. C'est bien de cet État qu'il est question lorsque qu'Habermas parle de l'État comme « pouvoir public » et lorsqu'il définit les attributs essentiels de ce dernier comme garant de « l'intérêt général, public, de tous les citoyens» (Habermas 2000:54). Selon Habermas, schématiquement, on peut présenter la structure de l'État-nation moderne telle qu'elle régit le processus démocratique sous quatre aspects :

En effet, dit-il, l'État moderne est né a)en tant qu'État administratif et fiscal, et b) en tant qu'État-nation territorial souverain, et il a pu se développer c) dans le cadre de l'État-nation d) pour prendre la forme d'un État de droit et d’un État social (Habermas 2000:4).

Présentée comme modèle archétypal, cette conception de l'État a connu deux siècles de succès au point où Habermas n'a pas manqué de souligner que « les démocraties, au sens occidental, ont dans la plupart des cas pris la forme de l'État-nation ». Mais seulement, en publiant à la fin des années 1990 Après l'État-nation. Une nouvelle constellation politique, Habermas fait ce constat quelque peu acrimonieux : « Depuis les années 1970, la forme d'institutionnalisation mise en œuvre par l'État-nation subit de plus en plus la pression de la mondialisation » (Habermas 2000). Que suggère ce constat ?

En réalité, le capitalisme, qui a été à l'origine de «la sphère publique bourgeoise » et, par conséquent, de l'espace public, a changé de visage. Dérégulé, diffus, acentrique, asymétrique et multiforme, le néo-capitalisme bourgeois est rentré dans sa dernière phase (Jameson 1991), celle dite de la mondialisation. S'arrachant des monopoles et des cadres institutionnels traditionnels, la nouvelle perspective du capitalisme planétarisé bouleverse tous les repères et les référents. Induit par des phénomènes tels que le mouvement des capitaux, le déplacement continu des populations, la circulation des images et des idées à travers la planète grâce à l'innovation technologique, la consommation de masse, la publicité, le marketing et la globalisation d'une culture idéal-type, ${ }^{2}$ le néo-capitalisme ouvre et inaugure un nouveau segment historique dans lequel la citoyenneté, la nationalité, le territoire, le local, devenus plus que jamais mobiles, imposent un découplage des espaces, des temporalités et une complexification de notre inscription dans un territoire assigné. Il devient de plus en plus difficile de parler de territoires nationaux comme des entités ou des réalités physiques, naturelles, figées et immuables. 
Avec le processus de la globalisation du capital, la souveraineté de l'État n'est plus conçue comme une et indivisible, mais elle est partagée avec des acteurs internationaux, transnationaux, infranationaux, voire non étatiques. Cela implique que les frontières territoriales et politiques sont de plus en plus perméables, rendant caduque l'idée d'une représentation de la souveraineté et de la citoyenneté assignée à un espace territorial défini et régi par des mécanismes institutionnels rigides. Dans un tel contexte, l'espace public, lieu de référence, de négociation, de désignation et d'assignation de la citoyenneté et de construction d'un État démocratique se trouve profondément altéré.

L'État-nation qui constituait jusque-là l'ancrage privilégié d'une citoyenneté stable prise dans un projet collectif et exclusif d'identification et d'authentification est défondé et irrémédiablement désubstantialisé. Les nouvelles citoyennetés se produisent dans un réseau ${ }^{3}$ de relations nomadiques, apatrides, formant ainsi des "sphères publiques d'exilés " (Appadurai 2001:11). Nous devenons tous, au moins virtuellement, nomades et sédentaires à la fois. Le territoire n'est plus géographiquement ou anthropologiquement situé. Il se (re)invente continuellement. Les groupes aux identités multiples, flexibles, mobiles ou complexes produisent leur local, leur propre territoire dans un monde de plus en plus déterritorialisé. La globalisation du capital nous impose donc la « déterritorialisation ».

Popularisé au début des années 1970 par Gilles Deleuze et Félix Guattari, qui s'étaient déjà préoccupés des dynamiques historiques du capitalisme dans leur entreprise philosophique, le concept de déterritorialisation est au cœur de la mondialisation actuelle. Concept opératoire et mobilisant, la déterritorialisation reconfigure la géomorphologie et la géopolitique mondiales. Elle permet de saisir la géopolitique comme une «micropolitique » dans laquelle l'être se donne comme spatialement et socialement " segmentarisé », arpentant permanemment les « lignes de fuites » et les zones de démarcation dans un mouvement continu de délocalisation et de désertion. La déterritorialisation interpelle ainsi sur la question de la distribution et de la gestion de l'espace et, par incidence, sur une nouvelle définition de la géographie. Elle implique l'idée selon laquelle la géographie n'est plus nécessairement l'étude de l'enracinement séculaire d'une communauté humaine dans un milieu naturel, mais une analyse de flux, de réseaux, de paysages mouvants dans « un pur devenir illimité ». La déterritorialisation impose dans ce sens une « pensée de l'espace » ou « une spatialisation de la pensée » (Antonioli 2003:8). Il s'agit de mener une réflexion « géophilosophique » sur la condition destinale du sujet humain dans la société néocapitaliste déréglementée et protéiforme. 
La congruence qui se crée entre la géographie et la philosophie et que Deleuze et Guattari ramassent sous la coupe conceptuelle de " géophilosophie » (Deleuze et Guattari 2005:82) devient ainsi un outil théorique définissant un cadre d'analyse qui permet de voir comment notre compréhension du monde contemporain, charrié par la globalisation du capital, appelle une redéfinition, voire une réévaluation des notions d'espace et de territoire - physique, mental, social, politique, économique, culturel, symbolique, etc. - dans la construction de nouvelles citoyennetés postnationales ou post-étatiques. Toute la question reste de savoir comment une telle problématique prend corps dans la pensée deleuzienne.

\section{Géophilosophie et nouvelle image de la pensée : une ontologie du nomadisme}

Le concept de « géophilosophie » fait l'objet d'un chapitre dans Qu'est-ce que la philosophie de Gilles Deleuze et Félix Guattari. Dans ce chapitre, les deux auteurs entreprennent une « reconfiguration territoriale de la pensée » (Deleuze et Guattari 2005:82). L'entreprise prend comme présupposé philosophique : la « déconstruction » de la métaphysique traditionnelle. Cette dernière est considérée, à tous les égards, comme l'assise fondamentale de la philosophie occidentale. Dans sa théorie de la connaissance, cette métaphysique a souvent établi des rapports particuliers entre le sujet et l'objet, le souci étant de trouver un sol stable et inamovible sur lequel la connaissance peut s'édifier. La connaissance est alors assimilée à la représentation adéquate de la chose à la pensée. Connaître, c'est se représenter adéquatement ce qui est en dehors de l'esprit et établir une identité entre le sujet pensant et l'objet pensé. A partir de là, comprendre ce qu'est la connaissance et ce qui la rend possible, ce n'est rien d'autre que comprendre comment l'esprit est capable de construire de telles représentations. La représentation devient ainsi le lieu objectif ou électif de la pensée, son fondement (Deleuze 1968:351). C'est bien dans ce sens que Deleuze écrit que "fonder, c'est toujours se représenter » et le fondement n'est rien d'autre que l'opération de la raison. Voilà ce que nous enseignent les philosophies du sujet associées aux noms de Descartes, Locke, Kant ou Hegel.

Pour Deleuze et Guattari, il est question d'opérer un changement de perspective philosophique. Ce changement de direction défend l'idée selon laquelle « penser n'est ni un fil tendu entre le sujet et l'objet ni une révolution de l'un autour de l'autre » : « penser se fait plutôt dans le rapport du territoire et de la terre » (Deleuze et Guattari 2005:82). Retrouver un territoire à la pensée qui échappe à toute emprise de la raison, tel est l'objectif que s'assignent Deleuze et Guattari. Comme postulat de base à cette entreprise, ils admettent que la terre de la pensée est mobile, non situable, non localisable. 
Cela implique que la pensée échappe à tout fondement, à toute territorialité fixe, défie tout ancrage. Le parti pris de Deleuze et de Guattari est de poser les bases d'une « ontologie faible ", ${ }^{4} \mathrm{~d}$ 'une pensée non ontologique qui tente de désancrer les certitudes et d'ébranler les «absolus » référentiels. Mais cette vaste entreprise de défondation et de désubstantialisation de l'être n'a de sens que pris dans le mouvement d'ensemble de la pensée de Deleuze. L'un des moments essentiels de cette pensée est «la théorie de la multiplicité », dont les premières intuitions sont présentes dans son ouvrage Différence et répétition.

\section{Différence et répétition ou comment penser la multiplicité}

Différence et répétition est le titre d'un des ouvrages essentiels de G. Deleuze. Cet ouvrage marque un point crucial dans l'évolution de sa pensée. En réalité, il peut être présenté comme le dernier maillon d'une chaîne d'ouvrages d'aspect plus classique de l'histoire de la philosophie que sont ses monographies sur David Hume, Baruch Spinoza, Emmanuel Kant, Friedrich Nietzsche, Henri Bergson. Différence et répétition se présente comme une synthèse des acquis successifs qui ont contribué à former et à forger la pensée personnelle de Deleuze. Dans cet ouvrage, Deleuze propose une conception du monde inédite en ce sens qu'elle nie toute stabilité et exige de sortir des cadres habituels de la pensée. C'est donc d'une nouvelle ontologie qu'il est question. Que suggère l'ontologie deleuzienne ?

D'abord, l'idée que rien ne se répète jamais vraiment à l'identique, au même. Deleuze prolonge ici la vielle doctrine héraclitéenne selon laquelle " on ne peut pas entrer deux fois dans le même fleuve ", la raison étant que l'eau ne cessant jamais de s'écouler est chaque fois différente bien que le nom du fleuve reste identique. Cette vision héraclitéenne laisse penser que c'est finalement la nature entière qui s'écoule de la sorte en se cristallisant provisoirement dans tels ou tels phénomènes. C'est dans ce canal conceptuel que Deleuze admet également que tout flue dans un " devenir " perpétuel, toute impression de stabilité n'étant que pure illusion. ${ }^{5}$ Dans ce que, de façon superficielle, nous croyons voir se répéter l'identique, le même ou le semblable, qui «fourmillent» en fait d'infimes différences qui font de chaque « retour ${ }^{6}$ Deleuze a en arrière plan de sa pensée un événement toujours nouveau et irréductible à ce qui l'a précédé.

Substantiellement, Deleuze affirme, contre toute la métaphysique traditionnelle pour qui la stabilité et la permanence sont des indices de la réalité d'une chose, que l'être se dit ou s'énonce en termes de " devenir ». Ce retour à la problématique antique sur la question de l'être et du devenir, Deleuze la met en relation avec nombre d'oeuvres modernes. Ainsi, selon lui, il n’y a jamais de répétition que de différence. 
A partir de cette nouvelle ontologie, Deleuze va se livrer à une critique violente de notre représentation courante de la réalité. Puisant abondamment dans le nietzschéisme et l'intuitionnisme bergsonien, Deleuze montre que notre représentation de la réalité est asservie au besoin d'utilité et d'efficacité nécessaire à la conduite régulière de notre vie en société. A cause de ce besoin, nous allons directement à l'essentiel, c'est-à-dire à ce qui peut être l'objet d'une connaissance stable. Ainsi, dans l'appréhension d'un phénomène par exemple, en nous épargnant de la considération superflue des contingences et des multiples différences accidentelles qu'elle recèle, nous ignorons ce que nous ne pouvons reconnaître immédiatement, ou ramenons si possible l'inconnu à du déjà connu, le nouveau à l'ancien. Selon cette optique, la différence se trouve "crucifiée » sur les quatre carcans qui constituent la théorie de la représentation : c'est toujours par rapport à une identité conçue, à une analogie jugée, à une opposition imaginée, à une similitude perçue que la différence devient objet de représentation (Deleuze 1968:339).

Toute anomalie, toute différence singulière, devient dans cette perspective une déviation par rapport à un modèle, une anormalité lorsqu'elle excède le cadre institué par le jugement normatif. L'anomalie singulière, qui est nécessairement une « différence ", n'est plus abordée pour elle-même, mais pour être comprise par une pensée cherchant à se la représenter. Cette anomalie est donc contrainte de passer le filtre de la ressemblance et de l'équivalence. De ce point de vue, nous ne connaissons des choses que des généralités par lesquelles elles se ressemblent : les essences.

Or ce qui domine la pensée de Deleuze, c'est précisément l'idée suivante : malgré toutes les déterminations fixes qu'on peut reconnaître à l'homme et à la nature, ces déterminations sont constamment traversées par des anomalies sauvages « immaîtrisables », non conceptualisables et inclassables selon les critères de la représentation. (Deleuze 1968:339). C'est bien cette idée qu'exprime Deleuze lorsqu'il écrit : « Mais il apparaît que de pures disparates forment ou bien l'au-delà céleste d'un entendement divin inaccessible à notre pensée représentative, ou bien l'en deçà infernal, insondable pour nous, d'un océan de la dissemblance » (Deleuze 1968:339). Le grand mérite que Deleuze reconnaît ainsi aux sciences humaines et plus particulièrement au structuralisme, c'est de dissoudre l'homme dans une multiplicité de structures relationnelles et accidentelles. C'est ce que signifie par exemple le thème de « la fin de l'homme » chez Michel Foucault, développé dans Les mots et les choses.

Selon Deleuze, philosophiquement, l'accès à ces différences pures ne peut s'opérer qu'en sortant radicalement du domaine de la représentation vers ce qu'il nomme « subreprésentatif » (Deleuze 1968:344). Pour cela, le philosophe doit court-circuiter l'activité de l'entendement et suspendre 
l'application des catégories habituelles que cet entendement produit spontanément pour la construction des représentations. Mais cette passivité volontaire ne vise qu'à permettre à la pensée du philosophe de se faire activer par autre chose : la rencontre violente et fortuite de l'étrangeté et de la nouveauté. Contre l'idéal de recognition auquel le rationalisme nous a accoutumés, il s'agit donc de se confronter à l’inconnu, à l'impensé, à ce qui est inexplicable dans le cadre de nos représentations communes. En effet, les rationalistes sont restés attachés au postulat de la vérité comme objet principal de leur recherche. Cependant, le caractère perpétuellement changeant des apparences derrière lesquelles ne se cache plus aucune identité mais des simulacres ${ }^{7}$ rend obsolète une telle recherche.

Cette nouvelle théorie de la connaissance, qui se pose en s'opposant au «bon sens » et au sens commun, affirme le principe de la déficience de l'être, de son atomisation, de sa fragmentation, de sa molécularisation, de sa fluidification, de sa multiplicité, de son altérabilité. Il est en effet remarquable que la dissolution du sujet humain dans la multiplicité des relations accidentelles qui le structurent ne doit pas, selon Deleuze, être simplement affirmée théoriquement, mais être vécue expérimentalement. Dans cette perspective existentielle, la différence ontologique prend la figure de l'intensité. L'intensité est cette force mobile qui anime la multiplicité et pose les rapports différentiels. Elle est l'être du sensible où le différent se rapporte au différent, le pouvoir d'affirmer la différence (Deleuze 1968:342). Principe d'ébranlement et d'écartèlement de l'être, l'intensité pose que l'on doit arrêter de se considérer comme un sujet substantiel, rationnel, stable, conscient de soi et identique à soi-même et qui se représente les objets eux-mêmes substantiels. C'est cette affirmation de la vie comme expérience volatile, fugitive et évanescente qui conditionne la territorialité de la nouvelle philosophie deleuzienne.

\section{Territorialité et nouvelle philosophie}

Quel statut Deleuze attribue-t-il à la philosophie aujourd'hui ? Il en donne lui-même une réponse éloquente : « la philosophie est une géo-philosophie, exactement comme l'histoire est une géo-histoire » (Deleuze et Guattari 2005:91). Cette réponse indique que la pensée devra de plus en plus s'ouvrir aux espaces, aux territoires, reconnaître sa dimension essentielle d'espacement et ne plus se limiter à une médiation sur son histoire et l'histoire des concepts comme point d'ancrage.

Deleuze marque une césure nette avec la philosophie classique qui, orientant la pensée vers la recherche de la vérité, voit en elle l'œuvre d'un sujet constitué qui connaît des objets et qui opère à l'aide de facultés distinctes qui ont chacune un territoire spécifique : sensibilité, imagination ou raison. 
Il est question pour Deleuze de tracer une histoire de la philosophie atypique et ironique qui privilégie les penseurs et les courants qui ont mis en question cette répartition des territoires de la pensée. On peut comprendre le grand attrait que Deleuze a vis-à-vis des pensées nietzschéennes, bergsoniennes ou heideggériennes, pensées dont l'objectif essentiel est de défonder la raison comme territoire naturel de la philosophie. Le nietzschéisme reste ainsi sa principale source d'inspiration.

En adoptant un style aphoristique, Nietzsche manifestait déjà sa désapprobation pour tout ce qui était donné comme entreprise systématique ${ }^{8}$ ou conceptuelle du réel. Pour Nietzsche, contrairement à la métaphysique traditionnelle qui posait le concept comme impersonnel, universel et principe général de toute chose, le concept n'existe pas en soi. Il est une fabrication des catégories de notre sensibilité. Nous le considérons abusivement comme s'il représente l'essence des choses.

C'est cette perspective que Deleuze adopte dans son ouvrage Qu'est-ce que la philosophie ? Si la philosophie est définie par Kant comme une « connaissance rationnelle par concepts » (Kant 1945:493), le concept, pour Deleuze, n'est qu'une pure création renvoyant au sensible et à l'art : « Les concepts, dit-il, ne nous attendent pas tout faits, comme des corps célestes. Il n’y a pas de ciel pour les concepts. Ils doivent être inventés, fabriqués ou plutôt créés, et ne seraient rien sans la signature de ceux qui créent » (Deleuze et Guattari 2005:11). Les concepts s'identifient ainsi aux « affects » et aux " percepts ». Pour donner plus d'élan à son propos, Deleuze interpelle l'autorité de Nietzsche. Il peut dès lors écrire :

Car, suivant le verdict nietzschéen, vous ne connaîtrez rien par concept si vous ne les avez pas d'abord créés, c'est-à-dire construits dans une intuition qui leur est propre : un champ, un plan, un sol, qui ne se confond pas avec eux, mais abrite leurs germes et les personnages qui les cultivent (Deleuze et Guattari 2005:12).

A partir de cette base, Deleuze pose que tout concept est composé. Il n’y a pas de concept simple. C'est une « multiplicité » ayant toutes les composantes possibles dans le sens d'un chaos et d'un «pur devenir illimité ». Ainsi, établit Deleuze, « tout concept à un contour irrégulier, défini par le chiffre de ses composantes ». Cela étant, tout concept est une " hétérogenèse », c'est-à-dire une ordination de ses composantes par zones de voisinage. Toutefois, le concept, en subsumant ces composantes, les survole. Il est incorporel, bien qu'il s'incarne ou s'effectue dans les corps. Il ne se confond pas avec l'état de choses dans lequel il s'effectue. Ce qui l'anime, c'est l'intensité. C'est bien dans ce sens que Deleuze dit des concepts qu'ils sont « des centres de vibrations » (Deleuze et Guattari 2005:28). 
Cette nouvelle image du concept oblige la philosophie à opérer sa propre révolution pour retrouver un territoire autre que celui de la pensée rationnelle ou conceptuelle. Ce territoire, Deleuze le nomme « le plan d'immanence » (Deleuze et Guattari 2005:38). Selon Deleuze, le plan d'immanence n'est pas un concept pensé ni pensable, mais l'image de la pensée. L'image que la pensée se donne de ce qui signifie penser. De cette image de la pensée, voici ce qu'en dit Deleuze :

L'image de la pensée ne retient que ce que la pensée peut revendiquer en droit. La pensée revendique "seulement » le mouvement qui peut être porté à l'infini. Ce que la pensée revendique en droit, ce qu'elle sélectionne, c'est le mouvement infini ou le mouvement de l'infini (Deleuze et Guattari 2005:40).

Concrètement, Deleuze conçoit le plan d'immanence comme ce qu'il y a de non-conceptuel dans le concept, de non philosophique dans la philosophie. Le plan d'immanence est à la fois ce qui doit être pensé et ce qui ne peut être pensé. Ce serait le non pensé dans la pensée. Il est ce qui est intime à la pensée et en même temps son dehors, un dehors lointain que le monde extérieur, parce qu'il est un dedans plus profond que tout monde intérieur : c'est l'immanence, cette " trace » consubstantielle à toute pensée qui la pose comme différentielle et ruine toute autonomie.

Le nouveau « personnage philosophique » que défend Deleuze est donc celui qu'il nomme « l'idiot », en référence à René Descartes. L'idiot est un " personnage conceptuel », décentré, irrationnel, ironiste mais surtout esthétisant. C'est un créateur, mais un créateur qui veut faire de l'absurde la plus haute puissance de la pensée. Une pensée qui n'est plus assignée à un lieu, à un territoire. C'est une pensée mouvante, errante, vagabonde qui a comme réplique sociale « l'étranger, l'exclu, le migrant, le passant, l'autochtone, celui qui rentre dans son pays » (Deleuze et Guattari 2005:6566). Le philosophe a perdu son territoire, il est devenu un exilé. Il n'a plus de demeure. Il n'a plus un chez-soi. Les personnages conceptuels ont un rôle précis : «manifester les territoires, déterritorialisations et reterritorialisations absolues de la pensée ». Ils sont animés par un désir de changer constamment leur territoire (Deleuze et Guattari 2005:67). Le décentrement philosophique qu'opère Deleuze prend dès lors comme présupposé fondateur l'exil et postule une ontologie nomadique.

\section{Pour une pensée nomade}

Pour qu'il y ait de la pensée, il ne suffit pas de rester dans un territoire familier de la représentation toujours déjà balisé et reparti entre le Moi, l'Objet, le Concept. Il faut admettre l'extériorité ou l'étrangeté absolue de quelque chose ou de quelqu'un qui nous force à penser la contingence ou l'événement 
singulier d'une rencontre. Il faut penser à l'indiscernable, à l'indécidable, à l'impensé, bref au «non-sens » qui domine toute pensée. Cet impensé, Deleuze le conçoit comme ce qui est impersonnel, « pré-individuel ». Ce sont, selon lui, « les singularités, libres et nomades » en perpétuel déplacement (Deleuze 1969:166).

Le mot nomade apparaît au XVIe siècle. Selon Manola Antonioli, commentant la pensée de Deleuze, le mot nomade se dit des peuples et des sociétés dont le mode de vie comporte des déplacements continuels par opposition à « sédentaire » qui exprime l'idée d'une personne qui reste dans une région déterminée (Antonioli 2003:25). Ce qui intéresse Deleuze dans le terme de "nomadisme », ce n'est pas surtout l'idée de l'extrême mobilité ou de l'errance, mais la forme de distribution dans l'espace. Un espace qui devient, dans sa philosophie, mental, politique, esthétique. Le nomadisme devient la base même de l'ontologie deleuzienne (Antonioli 2003:25).

La pensée nomadique postule le principe de l'exil. Il s'agit alors de refuser de figer, de systématiser, de totaliser. Cette pensée cherche à chaque fois à désancrer les certitudes, restituant ainsi à la pensée ses droits à une pensée ouverte aux choses et aux flux du monde et toujours à la recherche d'une patrie. Il est question d'habituer le sujet humain à ne plus se croire à sa place « dans » le monde, de le déstabiliser, de le désarmer de la certitude qu'il habite quelque part, comme en son « lieu naturel » qui ne peut être à tout prendre que le vide interstitiel, l'être scindé qui le lie et le coupe à la fois du monde, de cultiver à la place l'impression d'être un étranger jeté dans le monde, un habitant sans habitat, un nomade sans tente (Dufrénois et Miquel 1996:25).

La pensée nomade impose une relation entre le sujet et l'espace. En effet, actionné par les intensités qui laissent échapper en lui des zones d'ombre, le sujet ne se donne plus que comme multiplicité et possibilité d'une imprévisibilité radicale. Le sujet n'est plus ancré dans des certitudes, mais il est « d'emblée multiple, pluriel, collectif, fruit d'agencements qui comportent beaucoup de termes hétérogènes ». Aussi la pensée d'un tel sujet s'installet-elle nécessairement en dehors de sa conscience. Son nouveau lieu d'élection est le monde des flux, des conjonctions et des disjonctions, des rencontres à chaque fois singulières et imprévisibles. La conséquence devient dès lors que le dehors s'installe dans la pensée à travers l'extériorité des espaces et des lieux. Or, le plus souvent, ces lieux et ces espaces sont quadrillés ou striés par l'État et ses appareils de contrôle.

C'est dans cette veine qu'Antonioli peut établir un rapport consubstantiel entre la pensée et l'État. Elle écrit à ce sujet :

La pensée implique donc toujours des formes de distributions. L'histoire de la philosophie occidentale a su imposer progressivement une distribution qui présuppose un partage du distribué, une distribution sédentaire, dans 
la pensée et l'État (distribution qui est toujours solidaire d'un État organisé et centralisé, " pensée-État, toujours chargée d'une dimension politique lourde de conséquence) (Antonioli 2003:25-26).

La pensée de Hegel illustre en quelque sorte cet état de chose. Chez Hegel, l'État est le rationnel en soi et pour soi. Il est l'expression achevée d'une pensée qui a réalisé son concept, c'est-à-dire qui manifeste sa liberté absolue. L'homme ne développe pleinement sa connaissance comme sa liberté que comme citoyen d'un État. Il y a une identité manifeste entre la pensée et l'État.

Ainsi, si depuis la Grèce antique, la pensée a toujours été associée à un territoire, à une « polis », Deleuze propose une déterritorialisation dans laquelle on se distribue dans un espace ouvert, illimité ou du moins sans limite territoriale précise. C'est une distribution « nomadique » sans enclos ni mesure, où l'être se retrouve dans l'errance et l'hybridisme, longeant des espaces lisses et désertiques. Il s'agit de l'escapade du sujet dans des zones d'indiscernabilité, dans des territoires neutres, des vides interstitiels où les frontières entre individus s'estompent jusqu'à des «flux moléculaires ». La pensée nomade devient une politique nomade avec, comme thème majeur, la déterritorialisation.

\section{Déterritorialisation, schizophrénie et histoire du capitalisme}

En publiant en 1972 L'Anti-Oedipe. Capitalisme et schizophrénie, la pensée de Deleuze connaît un tournant décisif. Partant des intuitions métaphysiques qui tentaient de saisir l'être dans la multiplicité et le mouvement, cette pensée va s'intéresser au champ politique. Précisément, elle va tenter de définir une ontologie du politique. L'Anti-Oedipe, dans la sphère politique, se présente comme un véritable bréviaire de contestation, annonçant « une révolution anti-capitaliste ». L'ouvrage écrit collectivement par Deleuze et Guattari se présente comme un harcèlement incessant du capitalisme et des pouvoirs établis. La question fondamentale qui taraude l'esprit de Deleuze et de Guattari peut se résumer en ces termes qu'énonce Philippe Mengue :

Le capitalisme, qui ne peut subsister sans révolutionner en permanence ses forces et les rapports de production, est-il bien, lui aussi, une puissance « établie » ? ou bien, tout au contraire, une puissance de déterritorialisation sans précédent, débordant et contournant par surprise tout ce qui est stable ainsi que les projets des révolutionnaires professionnels les plus aguerris ? (Mengue 2003:15).

Pour donner une réponse à cette question, il faut suivre la structure logique de la pensée deleuzienne élaborée dans cet ouvrage. L'Anti-Oedipe peut schématiquement se comprendre à partir de deux grilles de lecture complémentaires : une critique de la psychanalyse au nom de la schizophrénie et une critique des dynamiques et de l'histoire du capitalisme, tout ceci ayant 
en creux une volonté d'associer le désir à la politique et formuler une politique du désir dans une « économie du désir inassouvi ».

\section{Des machines désirantes}

Le point de départ de l'analyse de Deleuze et de Guattari admet comme principe l'égalité entre la production sociale et la production désirante. Chez ces auteurs, le désir n'est plus l'expression d'un manque, mais une activité incessante de production qui travaille perpétuellement, de l'intérieur, les sociétés et l'histoire humaines. Le travail de production associe généralement trois réalités : l’homme, la nature et la machine. Mais chez Deleuze, « il n’y a plus ni homme ni nature, mais uniquement un processus qui produit l'un dans l'autre et couple les machines » (Deleuze et Guattari 1975:8). Partout on ne rencontre plus que des "machines désirantes », des "machines productrices » ou des "machines schizophrènes ». Ces «machines » ont pour fonction de couper les «flux » et de les (re)connecter sur d'autres « flux » dans une activité incessante et impersonnelle.

L'énergie désirante ne peut pas être attribuée au seul sujet ou enfermée dans la dimension sociale de la famille, séparée de l'économique et du politique. C'est bien dans cette optique que Deleuze et Guattari vont polémiquer avec la psychanalyse freudienne qui suppose, à travers le complexe oedipien, « une fantastique répression des machines désirantes ", leur enfermement dans la structure triangulaire du « moi-papa-maman ». La critique de la psychanalyse est portée au cœur de l'inconscient. En effet, pour Deleuze et Guattari, si la grande découverte de la psychanalyse fut celle de la production désirante, des productions de l'inconscient, cette découverte fut vite occultée par un nouvel idéalisme : à l'inconscient comme usine, on a substitué un théâtre antique : Edipe. Aux unités de production de l'inconscient, on a substitué la représentation (Deleuze et Guattari 1972:31).

Le péché de la psychanalyse est de vouloir comprendre scientifiquement et rationnellement les mécanismes de fonctionnement de l'inconscient, de les systématiser et surtout de les conceptualiser dans une théorie de la représentation. Pourtant, le désir comme production de l'inconscient échappe à cette entreprise systématique et conceptuelle. Le désir, soutiennent Deleuze et Guattari, ne manque de rien. Il ne manque pas son objet. C'est plutôt le sujet qui manque au désir, ou le désir qui manque de sujet fixe. Ils établissent que c'est tout le champ social qui est parcouru par le désir. Dans ce sens, « toute production sociale découle de la production désirante dans des conditions déterminées » (Deleuze et Guattari 1972:40).

Le reproche que Deleuze et Guattari font au corps social, au « socius », est celui de vouloir toujours " coder les flux du désir, les inscrire, les enregistrer, faire qu'aucun flux ne coule qui ne soit tamponné, canalisé, 
réglé ». Pourtant, les flux du désir sont par nature aléatoires et mutants. Ils échappent aux pouvoirs, certes, mais aussi à la raison. Voilà ce qui laisse penser qu'il y a une limite indépassable à l'intelligibilité rationnelle des flux. Les flux étant toujours fuyants, quelque chose échappe toujours à toute forme de prise ou de capture. On ne peut ni tout expliquer, ni tout prévoir, ni tout contrôler. Dans l'histoire de l'humanité, seule la société capitaliste est ancrée dans cette logique.

\section{Capitalisme, décodage des flux et schizophrénie}

Qu'est-ce qui fait la particularité du capitalisme par rapport à tous les modes de production qu'a connus l'histoire de l'humanité ? Deleuze et Guattari eux-mêmes donnent une réponse suffisamment claire à cette question :

La machine capitaliste, en tant qu'elle s'établit sur les ruines plus ou moins lointaines d'un État despotique, se trouve dans une situation toute nouvelle : le décodage et la déterritorialisation des flux. Cette situation, le capitalisme ne l'affronte pas du dehors, puisqu'il en vit, y trouve à la fois sa condition et sa matière et l'impose avec toute sa violence. Sa production et sa répression souveraine ne peuvent s’exercer qu’à ce prix (Deleuze et Guattari 1972:41).

Que comprendre de ces propos ? Selon Deleuze et Guattari, le capitalisme représente la plus formidable puissance de décodage des flux. Il est le « seul » agencement qui puisse se construire sur des «flux décodés », qui « libère les flux de désir ». Ce qui fait sa particularité, c’est le décodage généralisé des flux et leur conjonction : « la nouvelle déterritorialisation massive, la conjonction des flux déterritorialisés » (Deleuze et Guattari 1972:265-266). Deleuze et Guattari sont encore marxistes à ce niveau. Car, comme K. Marx, ils admettent que l'industrie capitaliste est éminemment révolutionnaire. Le capitalisme est révolutionnaire en ce sens qu’il

Fait tout vaciller, même le mieux établi ! Il déchaîne une puissance de production inégalée jusque-là, il lance toujours plus loin des flux de production, de biens de service, de travailleurs, mais aussi des flux de connaissance, de machines (...). Il décode, il déterritorialise, lance des flux de production, de discours, de pensée, d’art en tous les sens : Quelle liberté ! Quelle inventivité ! Quelle énergie, quelle audace ! Quelle émancipation par rapport aux codes anciens, aux rapports sociaux figés, engourdis dans les traditions ( «immobilisé dans la rouille »), quelle fluidification ! (Mengue 200:113).

Voilà ce qui fascine Deleuze et Guattari dans le capitalisme. C’est cette apologie qui est faite dans l'Anti-CEdipe. Le capitalisme tend à un seuil de décodage qui défait le «socius » au profit d'un corps sans organe et qui, sur ce corps, libère les flux de désirs dans un champ déterritorialisé. Le devenir de 
l'humanité se joue dans les désirs totalement libérés. C'est bien dans ce sens que Deleuze et Guattari ont beau jeu de dire que « le devenir universel, c'està-dire l'Histoire, a pour fin la production désirante dans sa libération à l'égard de la production sociale ». Cette libération du désir, Deleuze et Guattari la nomment : «processus schizophrénique ».

Pour nos deux auteurs français, la schizophrénie est la réalité même de la production désirante dans toute son authenticité, prise dans son absoluité. Elle consiste à « faire passer des flux à l'état libre sur un corps sans organe désocialisé » (Deleuze et Guattari 1972:292). Avec le corps sans organe, il s'agit de remplacer la distribution des organes dans l'organisme par la distribution anarchique du désir dans la surface d'un corps plein traversé par des flux qui ne s'arrêtent pas et qui ne s'expriment pas à l'aide d'un organe unique. Le corps sans organe est une surface d'enregistrement où toutes les synthèses fragmentaires et partielles viennent s'inscrire.

Si le capitalisme apparaît comme la limite de toutes sociétés, ce qu'elles n’ont cessé de conjurer, en tant qu'il opère le décodage généralisé des flux de désirs (Deleuze et Guattari 1972:180), Deleuze et Guattari nous mettent en garde : «ce serait une grande erreur, pensent-ils, d'identifier les flux capitalistes et les flux schizophréniques, sous le thème général d'un décodage des flux du désir » (Deleuze et Guattari 1972:291) ; « la schizophrénie n’est donc pas l'identité du capitalisme, mais au contraire sa différence, son écart et sa mort » (Deleuze et Guattari 1972:293).

Pour Deleuze et Guattari, le schéma de formation du capitalisme le contraint à contrarier le procès de déterritorialisation sur lequel il repose. Le capitalisme " axiomatise » les flux décodés et les re-territorialise. C'est ce qu'exprime Mengue lorsque, analysant la pensée de Deleuze, il écrit que « Pour se maintenir, et éviter toute autodestruction, le capitalisme a besoin de contrôler, d'arrêter la déterritorialisation des flux qu'il lance, de rabattre l'instance de production qu'il ne cesse de relancer sur des terres artificielles, archaïques et, ainsi, coincer, ligaturer le processus de la production sociale et désirante » (Mengue 2003:117). La schizophrénie au contraire libère l'écoulement des flux sans aucune régulation. C’est ce pas décisif que le capitalisme ne parvient pas encore à franchir. C'est l'acrimonie que manifestent Deleuze et Guattari. Pour eux, « le capitalisme n'est pas ce flux d'énergie entièrement libre, la production purement désirante émancipée, qu'il semble être » (Mengue 2003:115). A cette requête, le capitalisme postmoderne semble donner un avis très favorable.

\section{Capitalisme postmoderne et économie du désir inassouvi}

Pour Deleuze et Guattari, rien n'est préalable au désir et à ses libres agencements. Le plaisir, la jouissance ne sont pas épanouissement mais connexion, branchement des hétérogènes, capture et détournement. Mais le 
capitalisme, malgré sa capacité de déterritorialisation, partage toujours avec les formations sociales passées le trait d'être répressif à l'égard de la production désirante. C'est le grand regret qu'expriment Deleuze et Guattari quand ils disent que le capitalisme, comme aucune autre formation sociale du passé, n’a pu supporter le libre écoulement des flux sur le « corps-sansorgane ». Le corps sans organe, comme surface libidinal ou plan d'agencement du désir, a toujours jusqu'ici été pris dans une machine sociale déterminée, un « socius comme plan d'organisation à l'échelle molaire ». Cette action de capture des flux du désir est l'œuvre de la famille et de l'oedipianisation qui, pour Deleuze et Guattari, sont consubstantielles à l'État.

Le rôle de l'État est de reterritorialiser le capitalisme. C'est ce qu'expriment Deleuze et Guattari quand ils posent qu'avec le rôle de l'État, les reterritorialisations, la nécessité de l'absorption de la plus-value (militarisme, publicité, gouvernement civil...), il y a une effusion généralisée de l'anti-production dans la production : l'anti-production dégage " un fantasme instinct de mort qui imprègne et écrase le désir » (Deleuze et Guattari 1972:312). Toutefois, si l'histoire universelle a pour fin la production désirante dans sa libération à l'égard de la production sociale, seul le capitalisme tend vers cette libération des flux de désir sur l'échelle des régimes répressifs. Le capitalisme représente le régime le plus libérateur qui soit à l'égard du désir et de ses agencements, le plus grand mouvement de déterritorialisation jamais vu. C'est cette particularité qui en garantit la permanence. C'est ce que note P. Mengue quand il écrit ceci :

Le capitalisme est inamovible dans l'histoire des sociétés humaines. On doit donc aller jusqu'à penser que le capitalisme, en tant qu'économie de libre marché, représente la logique économique, et comme tel il n'est plus, dans son principe, d'aucun régime. Il est au croisement de tous les flux de production et par nature il ne peut se maintenir qu'en se recréant à chaque fois. Il est donc toujours nouveau : néo-néo...capitalisme. Il ne cesse de s'inventer pour le meilleur comme pour le pire. Et chaque fois, il rencontre une forme sociopolitique qui le régule : au capitalisme des practiciens et des esclaves correspond la Polis ou la République romaine ; au capitalisme agraire et fluvial, l'État despotique et bureaucratique ; au capitalisme financier italien et hanséatique, les Cités libres de la Renaissance ; au capitalisme industriel des XVIIIe et XIXe siècles, la démocratie parlementaire, au capitalisme financier et boursier, la démocratie informatique des communications numériques (Mengue 2003:124).

Le capitalisme est, de par sa nature, révolution permanente. Si l'on est attaché à la libération maximale des flux, à la réalité des lignes de fuite, aux forces constamment renouvelées de déterritorialisation, que peut-on trouver de mieux que le capitalisme ? Dans sa dernière phase, celle dite postmoderne, la 
déterritorialisation se donne comme phénomène de massification des flux des gens apatrides, déracinés, « coulant » sur des territoires nationaux, sapant leur tradition et enracinement psychique, social, politique, économique, culturel ou symbolique. Dès lors, ils sont démunis de toute protection juridique et d'appartenance citoyenne. N'est-ce pas un tel ultralibéralisme qu'ont souhaité Deleuze et Guattari ? La dynamique actuelle du capitalisme réalise bien les vœux deleuziens : libérer totalement les flux de désir dans une économie du désir inassouvi.

Toute la « théorie politique » de Deleuze tente d'établir la non nécessité des états des choses, leur contingence, leur mobilité historique et leur possible transformation permanente. Il faut contester et déstabiliser les pouvoirs et les ordres établis, ce qu'il appelle par ailleurs les «segments durs » ou " molaire » et laisser proliférer la logique microscopique ou microbienne des « segmentarités fines ». Ces dernières échappent à toute mise en forme macroscopique et à toute classification rigide : c'est l'avènement de la «micro-politique ». Selon Deleuze, la seule chance qui nous reste, notre unique chance est dans le «micro ». C'est cette confidence qu'il fait à Antonio Negri lorsqu'il dit : « croire au monde, c'est aussi bien susciter des événements même petits qui échappent au contrôle, ou faire naître de nouveaux espaces-temps, même de surface ou de volume réduit. »

\section{Micro-politique et nouvelles citoyennetés dans la mondialisation du capitalisme postmoderne}

Deleuze considère dans un premier temps la micro-politique comme une action ou un événement de petite dimension visant un intérêt local, sectoriel et impliquant des groupes de petite taille, des individus, des singularités. Le concept de micro-politique est une analyse du moléculaire ou du " segmentaire ». C’est bien cette idée qu'exposent Deleuze et Guattari dans leur ouvrage collectif Mille Plateaux. Capitalisme et schizophrénie 2. L'une des idées capitales défendues dans cet ouvrage est que notre vécu est « segmentarisé » spatialement et socialement dans toutes nos activités.

La notion de segmentarité est empruntée à l'ethnologie où elle servait comme outil de description des sociétés dont l'organisation excluait la forme centralisée et unifiée de l'État. Dans l'ethnologie, le segment est un principe de souplesse, le nom donné à la forme d'organisation qui résulte de la combinaison des codes et territoires, des lignages claniques et des territorialités tribales, une segmentation en acte et en devenir qui ne cesse de se réorganiser par des combinaisons toujours nouvelles et itinérantes. Deleuze et Guattari refusent pourtant la dichotomie qu'on veut établir entre le central et le segmentaire. Pour eux : 
Le système politique moderne est un tout global unifié et unifiant, mais parce qu'il implique un ensemble des sous-systèmes juxtaposés, imbriqués, ordonnés, si bien que l'analyse des décisions met à jour toutes sortes de cloisonnements, et de processus partiels qui ne prolongent pas les uns les autres sans décalages ou déplacements (Deleuze et Guattari 1972:225).

Chez Deleuze et Guattari, il existe deux segmentarités à la fois : l'une molaire ou dure et l'autre moléculaire ou souple. Ces deux segmentarités n'ont pas la même nature, mais elles coexistent toujours et ne cessent de passer l'une dans l'autre. Bref, pour eux, « tout est politique, mais toute politique est à la fois macropolitique ou micropolitique. » Deleuze et Guattari ne sont pas ici éloignés des vues de Michel Foucault qui, dans Surveiller et punir, montre que l'analyse politique ne peut pas se limiter aux « segments durs » constitués par les centres visibles de pouvoir (État, armée, école, hôpital, prison, église, etc.), mais tout centre de pouvoir visible n'est que le lieu où rentrent en résonance toutes sortes de micropouvoir, des devenirs imperceptibles où le pouvoir n'existe qu'à l'état diffus, dispersé, démultiplié, miniaturisé. Tout centre de pouvoir, note Manola Antonioli, «a sa zone de puissance qu'il contrôle, sa zone d'indiscernabilité faite de micropouvoir et de flux, sa zone d'impuissance faite de flux qu'elle ne peut ni contrôler ni déterminer " (Antonioli 2003:112).

C'est bien la logique de fonctionnement de la mondialisation actuelle. L'État-nation, tel qu'il a été construit par la modernité européenne, présuppose un lien solidaire et défini entre un peuple, un appareil politique centralisé, un territoire dont les frontières peuvent être bien définies et une unité politique homogène et souveraine. Mais avec la déterritorialisation du capital, il doit faire face à d'autres unités souveraines à l'extérieur comme à l'intérieur de ses frontières.

\section{Néocapitalisme et "sphère publique des exilés"}

On a longtemps défini l'État comme un État-territoire. Mais aujourd'hui, on est confronté à de nouvelles formes de communautés politiques qui échappent à un territoire national, à une appartenance étatique et qui se mobilisent de façon locale et souple autour d'une cause, grâce, notamment, à Internet et à d'autres formes de réseaux. Selon Antonioli, reprenant les vues de Deleuze, le futur politique se joue dans l'équilibre permanent et mobile entre la sédentarisation et le nomadisme, la déterritorialisation et la reterritorialisation. A cet effet, Antonioli pense qu'aux marges ou en dehors d'une problématique institutionnelle, les nouvelles formes d'action politique microbiennes et souterraines sont tôt ou tard confrontées à une difficile alternative : s'isoler dans un excès de critique et une parcellisation de l'action qui risque de nuire 
à leur efficacité ou bien se doter d'une organisation réglée et institutionnelle et être réabsorbées par les structures étatiques auxquelles elles avaient essayé d'échapper (Antonioli 2003:58).

Cette opposition qui se joue entre l'homogénéisation et l’hétérogénéisation pousse l'anthropologue de la mondialisation, Arjun Appadurai, à établir un lien causal entre la dynamique révolutionnaire du capitalisme globalisé et l'attitude labile des populations exilées. Selon Appadurai, tandis que le capital international modifie ses besoins, tandis que les États-nations modifient leur politique vis-à-vis des populations réfugiées, ces groupes mouvants ne peuvent jamais, quels que soient leurs désirs, laisser leur imagination trop longtemps inactive. L'imagination, comme catégorie psychologique opératoire, joue un rôle moteur dans la construction de nouveaux territoires ethniques et culturels. Dans ces nouvelles localités, émergent des identités mixtes, complexes, flexibles, hybrides. Pour Appadurai, ceci n'est que la conséquence directe de nouvelles dynamiques initiées par les forces à la fois centrifuges et centripètes de la mondialisation.

La mondialisation actuelle s'offre à nous sous une double temporalité. D’une part, nous vivons une uniformisation accélérée du monde, grâce à une technologie croissante, devenue messianique. D'autre part, c'est plutôt une fragmentation accentuée du monde avec, notamment, des replis identitaires, sectaires, communautaires. Il existe dès lors un rapport constant entre les forces dites de progrès (innovation scientifique et NTICS) et les forces dites de régressions (retour à diverses sortes d'ancestralités). C'est ce que Régis Debray appelle : le principe de constance (Régis Debray 1996:156). Précisément à ce sujet, Debray écrit :

Chaque dispositif de déracinement et d'ouverture libère un mécanisme de contre-enracinement ou de clôture (...). Les territoires culturels que le progrès technique efface se recomposent dans l'imagination. Nous aboutissons à des patries apatrides (...). La demande de résidence secondaire, dans l'idée, compense l'évanescence des frontières primaires, dans le réel (Debray 1996:156).

Aux grandes avancées technologiques couplées aux flux migratoires toujours croissants répondent une évolution des imaginaires sociaux, un nouvel ordre d'instabilité dans la création des subjectivités modernes constitutifs de l'existence de nouvelles diasporas. Ces diasporas forment des publics enfermés dans leurs petites bulles ethnicisées. Les nouvelles sphères d'élection, de filiation ou d'affiliation sont de moins en moins dépendantes des territoires des États-nations.

Les constructions identitaires ne se font plus dans un « jeu permanent d'opposition entre soi et l'autre, entre l'intérieur et l'extérieur, mais plutôt dans la multiplication de sphères d'exilés » (Appadurai 2001:11), où des 
gens se réapproprient collectivement des récits et des images. Que font-ils de ces récits et de ces images ? Avec les récits et les images, les exilés peuvent s'inventer leur quotidien et leur localité. A une production rationalisée, expansionniste autant que centralisée, bruyante et spectaculaire correspond une autre production : celle-ci est rusée, dispersée et indocile. Elle s'insinue partout, silencieuse et quasi invisible, puisqu'elle ne se signale pas avec des produits propres, mais avec la manière d'employer et d'instrumentaliser les produits imposés par un ordre économique dominant (de Certeau 1980:XXXIX).

Les groupes migrent, se rassemblent dans des lieux nouveaux où ils peuvent reconstruire leur histoire et reconfigurer leur projet ethnique. Plus que jamais, les groupes ne sont plus étroitement territorialisés ni liés spatialement, ni dépourvus d'une conscience historique d'eux-mêmes, ni culturellement homogènes. Pour Appadurai, il existe un lien net entre le travail de l'imagination comme forme de résistance à la domination et l'émergence de solidarités globales susceptibles de donner naissance à un nouvel espace politique postnational.

La résistance se donne comme la diffusion de comportements résistants et singuliers. Antonio Negri, continuant la pensée d'Appadurai, pense que «si la résistance s'accumule, elle le fait de manière extensive, c'est-à-dire par la circulation, la mobilité, la fugue, l'exode, la désertion ». Et il ajoute : « il s'agit de multitudes qui résistent de manière diffuse et s'échappent des cages toujours plus étroites de la misère et du pouvoir » (Negri 2001:104). En réalité, dans l'ère postmoderne et postnationale, la forme la plus éminente de la rébellion est l'exode de l'obéissance. Cette multitude s'exile en se constituant, en se relocalisant, en se recontextualisant avec tout genre de mélange, de métissage, de créolisation. Une seule injonction domine donc le monde d'aujourd'hui :

Mélangez les races, et les cultures, constituez l'Orphée multicolore qui génèrera le commun à partir de l'humain. Détruisez toutes les barrières transcendantales qui empêchent le singulier de devenir commun et l'éternel d'innover : voilà ce qui signifie s'en aller en constituant (Negri 2001:210).

Selon Appadurai, les nouvelles localités et les politiques de la transfiguration (Benetta 1998:182) se forgent ainsi selon un double schéma :

- Le travail de l'imaginaire, à travers lequel est produite et nourrie la subjectivité locale, est un étonnant mélange de considérations fortement locales et fortement translocales.

- Les trois facteurs qui affectent le plus directement la production de la localité dans les temps postmodernes - l'État-nation, les flux diasporiques et les communautés électroniques ou virtuelles - sont 
articulés de façon variable, surprenante, parfois contradictoire, dépendant de l'environnement culturel, historique, écologique et de classe dans lequel ils se trouvent rassemblés. Cette variabilité est ellemême en partie un produit de la façon dont les "ethnoscapes" aujourd'hui interagissent avec les «financescapes", les " médiascapes », les « technoscapes » et les "idéoscapes ». ${ }^{9}$

En substance, la localité dans une situation où l'État-nation affronte des types particuliers de déstabilisations transnationales est avant tout une question de relation et de contexte plutôt que d'échelle et d'espace. Les formations culturelles globales formant des « postcultures », devenues plus que jamais fractales, sont dépourvues de frontières, de structures ou de régularités euclidiennes (Appadurai 2001:85). L'ethnie, dans la mondialisation actuelle, est rentrée dans la phase de la déterritorialisation et de l'hybridation. Le dépassement des frontières naturelles, la contamination des langages, le métissage des genres et des races, l'hybridation générale de l'être sont autant d'éléments constitutifs des nouvelles communautés délocalisées. Aujourd'hui, soutient Antonio Negri.

La transformation de l'outil, l'hégémonie productive des pratiques linguistiques et la formation d'un contexte biopolitique à l'intérieur de la subjectivité métamorphosent l'être humain et réalisent son hybridation en traversant toutes les frontières qui étaient fixées (Negri 2001:138).

La conséquence directe de la logique du monde postmoderne dans lequel nous sommes appelés à vivre est que l'émergence d'un ordre postnational qui défie tout souverainisme donne lieu non à un système formé d'unités homogènes (comme c'est le cas des États-nations), mais à un système fondé sur des relations entre des unités hétérogènes. Selon Appadurai, ce nouvel ordre va être confronté à un défi dont il croit donner une esquisse de solution. Il pose ceci :

Une telle hétérogénéité est-elle compatible avec un accord minimal sur les normes et les valeurs, sans pour autant adhérer strictement au contrat social libéral de l'Occident moderne ? On ne résoudra pas cette question décisive par un décret académique, mais par des négociations (recourrant autant au dialogue qu'à la violence) qui verront se confronter les mondes rêvés par ces intérêts et ces mouvements différents. A court terme, ce monde verra sans doute se développer des manifestations d'incivilités et de violence. A long terme, libérés des contraintes de la nation, nous pourrions trouver que la liberté culturelle et la justice se passent très bien de l'existence générale et uniforme de l'État-nation (Appadurai 2001:56-57).

Si tel est l'horizon indépassable vers lequel s'achemine notre monde, quel peut bien être encore le destin de la démocratie, cette forme de gouvernement 
considérée, à maints égards, comme le creuset indispensable de l'institutionnalisation de l'État républicain dans lequel la citoyenneté a été historiquement définie?

\section{Démocratie et opinion rhizomique}

La pensée de Deleuze peut fournir une toute autre conception de la démocratie en rupture avec l'idée traditionnelle qu'on s'est toujours faite d'elle. Pour comprendre cette rupture, il faut revenir aux intentions politiques de Deleuze. Selon lui, la lutte contre la métaphysique traditionnelle ne peut se faire que sur un fond de véritable « guérilla ». Cette thèse implique l’idée selon laquelle la politique doit s’émanciper des valeurs métaphysiques. Dès lors, la politique ainsi libérée est assujettie à la nouvelle image de la « pensée » dont les valeurs sont : l'intempestif ou l'intensité, la création, la nouveauté, le remarquable, l’événement et non plus le vrai ou le juste.

Toute la politique se ramène à la multiplicité, à la prolifération « rhizomatique » des comportements subversifs qui ne peuvent plus supporter un grand projet d'ensemble, une idéologie collective définissant une vision téléologique du monde ou un programme collectif de société. Toute politique, dans ce contexte, se donne dans le cadre d'une démocratie. Mais de quelle démocratie ? Une démocratie sans fondement, parce que chez Deleuze, elle est la seule logique possible dans le cas de la pluralité et de la rivalité des opinions. Le relativisme deleuzien pose que les principes démocratiques sont sans fondement, n’ont aucune vérité, encore moins une justification ultime. Rejoignant les vues de Nietzsche, il s’agit d'une démocratie esthétique, celle qui donne au sujet le libre choix d'adopter le mode de vie qu'il souhaite suivant les convenances de ses désirs. Il faut ici préserver la vitalité et les désirs essentiels de toute forme d'expression. Dans ce contexte, l'espace public devient le lieu d'une « créativité dispersée », l’espace où l'on doit ruser sans cesse, avec les règles ou les normes, pour connaître toutes les satisfactions nécessaires.

Ce point de vue est fortement soutenu par un néo-pragmatiste comme Richard Rorty qui, parlant de la société libérale postmoderne, dit ceci :

Le ciment social qui assure la cohésion d'une société libérale idéale (...) n’est guère plus qu'un consensus autour de l'idée que l'objet de l'organisation sociale est de laisser à chacun une chance d'auto-création au mieux de ses aptitudes et que cet objectif exige, outre la paix et la richesse, les libertés bourgeoises classiques. Cette conviction ne se fonderait sur une quelconque vision des fins humaines universellement partagées, des droits de l'homme, de la nature, de la rationalité, du Bien de l'homme ni d'aucune autre chose. Elle ne reposerait sur rien de plus profond que les faits historiques qui suggèrent que sans la protection de quelque chose comme les institutions 
de la société libérale bourgeoise, les gens seront moins capables d'assurer leur salut privé, de se créer une image d'eux-mêmes, de retisser leurs toiles de croyances et de désirs à la lumière de tous les individus ou livres nouveaux qu'ils peuvent rencontrer. Dans une société idéale de cette espèce, la discussion des affaires publiques tournera autour de la manière d'équilibrer les besoins de paix, de richesse et de liberté quand les conditions exigent que l'un de ces objectifs soit sacrifié à l'un des autres et de la façon d'égaliser les chances d'auto-création puis de laisser les individus, et eux seuls, exploiter leurs chances ou les négliger (Rorty 1989:126-127).

Rorty prône l'adhésion à une société dans laquelle rien n'est plus donné comme absolu. Tout devient contingent et toutes les perspectives sont possibles : c'est le règne du rhizome. Le rhizome devient le principe de la démocratie libérale postmoderne. Celui-ci pose la logique de la multiplicité des opinions et des avis. L'opinion n'est plus fixe, encore moins arrêtée. Elle est mobile et incertaine. Elle se fait fragile et flottante, sans ancrage défini et ultime. Elle est métaphorique et rieuse, car elle se moque de toute convention et de toute règle établie. Elle n'a pas de territoire, mais se trouve toujours dans un point de fuite, dans «l'entre-deux », dans un milieu qui n'a ni commencement, ni fin. En substance, l'opinion est toujours indéterminée, indécise et indiscernable. Elle suit la fluctuation de nos désirs toujours en quête d'une satisfaction jamais assouvie.

\section{Conclusion}

Le sujet que défend Deleuze se présente comme un individu déterritorialisé, fragmenté, désubstantialisé, flexible, mobile, sans identité définie : c’est un individu hybride et nomade. Il est pris dans ce que le penseur italien Gianni Vattimo, reprenant Martin Heidegger, appelle le « jeu de miroirs du monde ». Comme le reflet d'un miroir, ce sujet se saisit dans la fugacité, l'éphémère, la diversité, le contingent, le circonstanciel, le différent. Individu défondé, amnésique et sans repère fixe, il vit dans des interstices, constituant pour lui des « sphères publiques d'exilés ». Dans ces espaces se tissent des réseaux et des nœuds de relations apatrides, hybrides et décentrés. Dans un tel contexte, les qualités, les significations, et les fonctions que l'individu actualise dépendent de la position qu'il occupe. Il est en continuelle mutation. Son identité subit à tout instant de graves infractions à cause d'une économie du désir inassouvi. Cet individu n’a plus une identité racine, stable, permanente et garantie par une conscience autonome, une citoyenneté fixe. Mais il s'arcboute sur une identité rhizome, mobile, flexible, tactique et stratégique qui reconfigure à chaque fois son ancrage territorial et sa citoyenneté.

En rejetant la métaphysique traditionnelle et ses idéaux de fondement la raison, la vérité, l'autonomie du sujet, le progrès, l'histoire, l'universalité -, 
Deleuze promeut une herméneutique du sujet dans la logique du « souci de soi » foucaldien. Cette herméneutique coïncide avec les grands principes de la culture de consommation du dogme capitaliste. Les habitudes de consommation et la stratification des styles de vie sont, dans le capitalisme postmoderne, une fin d'accomplissement et d'épanouissement de soi. La diversité et la multiplicité des territoires, des cultures, des genres et de mode de vie ne sont pas une entrave à la grande entreprise capitaliste dans la mesure où les firmes industrielles et la structure économique doivent s'adapter à une demande changeante, fluctuante et de plus en plus segmentée. La fragmentation accentuée de l'être et du monde, leur flexibilité, leur modulation et le principe de déterritorialisation élargissent ainsi le marché de consommation à des fins stratégiques de rentabilité. L'espace public dans un tel contexte devient une sphère de multiplication des cellules ou des principautés consuméristes : c’est l'apothéose de la révolution consommatoire.

\section{Notes}

1. C'est ce qui caractérise le premier âge de l'espace public qu'Habermas nomme « la sphère publique structurée par la représentation ». La particularité de cette sphère, c'est qu'elle est un lieu où se déploie et se manifeste l'autorité publique à l'époque de l'autorité du roi. Autrement dit, c'est une sphère où sont rendus lisibles les rapports de force qui structurent la société d'Ancien Régime. Dans cette sphère, la société se laisse appréhender dans sa texture propre comme une société hiérarchisée sous l'emprise du monarque. La caractéristique principale de cette représentation, c'est le phénomène de cour lié à la monarchie absolue.

2. Des penseurs comme Francis Fukuyama ou Samuel P. Huntington pensent la mondialisation en termes d'occidentalisation du monde. La mondialisation est l'apothéose de la civilisation occidentale dont le trait caractéristique est ce que Huntington appelle « la culture de Davos ». C'est cette culture qu'il faut préserver de toute altération pouvant venir des fondamentalismes et des intégrismes.

3. Dans Après l'État-nation. Une nouvelle constellation politique, Habermas indique « qu'il s'agisse des voies de transports, des biens et des personnes, des flux de marchandises, du capital et de l'argent, du transfert électronique, du traitement des informations ou des circuits reliant l'homme à la technique et à la nature, le terme clé est désormais réseau.».

4. La notion d' " ontologie faible » est du penseur italien Gianni Vattimo. Dans son ouvrage La fin de la modernité. Nihilisme et herméneutique dans la culture postmoderne, Vattimo prolonge en quelque sorte les préoccupations essentielles de Deleuze. S'arc-boutant sur Nietzsche et Heidegger, dont le souci était de mettre en branle, voire de saper les fondements de la pensée occidentale ou de la pensée comme fondement du rationalisme, Vattimo pose 
le principe postmoderne de la dissolution de l'être. Selon lui, «tant que l'homme et l'être seront pensés métaphysiquement, c'est-à-dire sur un mode platonicien, et en termes de structures stables qui imposent à la pensée comme à l'existence le devoir de se « fonder » et de s'établir (par la logique et par l'éthique) dans le domaine du non-devenant, le tout se réfléchissant dans une mythologie de structures fortes étendues à tout le champ de l'expérience, la pensée ne pourra vivre en aucune façon la positivité de cette véritable ère post-métaphysique qu'est la post-modernité » (Vattimo 1987:17).

5. La philosophie occidentale se bâtit toujours sur un désir de fuir et de combler cette béance originaire de l'homme sur une quête éperdue de ce qui lui assurera un fondement suffisant. L'élaboration d'un système philosophique, dans cette tradition, n'est pas autre que la rationalisation de cette fuite, la constitution d'un monde cohérent comportant les fondements premiers à partir desquels tout devient clair, sans faille - l'erreur et le mal se trouvant eux-mêmes expliqués.

6. Deleuze a en arrière plan de sa pensée l'idée du mythe de l'éternel retour de Nietzsche. L'éternel retour, selon Nietzsche, n'est pas la pensée de l'identique, mais une pensée synthétique. Penser l'absolument différent qui réclame hors de la science un principe nouveau. Ce principe est celui de la reproduction du divers en tant que tel, celui de la répétition, de la différence. Dans sa monographie consacrée à Nietzsche, Deleuze fait remarquer que « nous ne comprenons pas l'éternel retour tant que nous en faisons une conséquence ou une application identique. Nous ne comprenons pas l'éternel retour en tant que nous ne l'opposons pas d'une certaine manière à l'identité. En d'autres termes, l'identité dans l'éternel retour ne désigne pas la nature de ce qui revient, mais au contraire le fait de revenir pour ce qui diffère ». L'éternel retour est donc toujours un événement (Deleuze 1988:55).

7. Ce concept joue un rôle décisif dans la pensée de Deleuze. Il est l'un des puzzles gagnants de l'entreprise de « renversement du platonisme », le platonisme étant le symbole de la métaphysique classique. En effet, pour Deleuze, le motif de la théorie des idées de Platon doit être recherché du côté d'une volonté de sélectionner, de trier. Il s'agit d'opérer des différences. Distinguer la « chose » même, c'est-à-dire l'idée de ses images, l'original de la copie, le modèle du simulacre. D’après Deleuze, il y a une différence entre la copie et le simulacre. La copie est une image douée de ressemblance, le simulacre une image sans ressemblance. Le simulacre ne peut donc être identifié au modèle. Si le simulacre a encore un modèle, c'est un autre modèle, un modèle de l'Autre dont découle une dissemblance intériorisée. Il y a dans le simulacre un devenir-fou, un devenir illimité, un devenir toujours autre, un devenir subversif, habile à esquiver l'égal, la limite, le même, le semblable (Deleuze 1969:297).

8. Nietzsche était un adversaire déclaré de tout système. Sur la question, voici les propos de Nietzsche que reprend George Lukacs : « Je me méfie de tous les penseurs systématiques, j'évite de les rencontrer. Vouloir bâtir un système, c’est manquer de loyauté » (Lukacs 1958:279). 
9. Le suffixe « scape », précise Appadurai, est tiré de landscape, « paysage ». Il permet de mettre en lumière les formes fluides, irrégulières des paysages sociaux. Ces paysages sont des «briques » de construction des "mondes imaginés", c’est-à-dire les multiples mondes constitués par les imaginaires historiquement situés de personnes et de groupes dispersés sur toute la planète. Les individus qui constituent les ethnoscapes sont : touristes, immigrants, réfugiés, exilés, travailleurs, etc.

\section{Bibliographie}

Antonioli, M., 2003, Géophilosophie de Deleuze et Guattari, Paris, L'Harmattan. Appadurai, A., 2000, Après le colonialisme. Les conséquences culturelles de la globalisation, traduit de l’Anglais (États-Unis) par Françoise Bouillot, Paris, Payot.

Barber, B., 1996, Djihad vs MC. World. Mondialisation et intégrisme contre la démocratie, Paris, Desclée de Brouwer.

Bennetta, J.-R., 1998, Black Paris, by the Board of Trustee of the University of Illinois, Manifactured in the United States of America.

Certeau (de), M., 1990, L'invention du quotidien 1. Arts de faire, Paris, Gallimard.

Corcuff, P., 2003, « Néo-capitalisme, individualisme, subjectivité en partant du Nouvel esprit du capitalisme et de l’Empire ». Colloque « Résistance au sujet - résistances du sujet ». Centre Culturel International de Cerisy-la-salle, 2131 juillet 2003.

Debray, R., 1996, « L’archaïsme postmoderne », in Les temps modernes, 51 année, n586, Jjanvier-février, pp. 155-163.

Deleuze, G., 1968, Différence et répétition, Paris, P.U.F.

Deleuze, G., 1969, Logique du sens, Paris, Les Editions de Minuit.

Deleuze, G., et Guattari, F., 1972, Mille Plateaux. Capitalisme et schizophrénie 2, Paris, Les Editions de Minuit.

Deleuze, G., et F. Guattari, 1975, L'anti-Oedipe. Capitalisme et schizophrénie, Paris, Les Editions de minuit.

Deleuze, G., 1988, Nietzsche et la philosophie, Paris, P.U.F.

Deleuze, G., et F. Guattari, 2005, Qu'est-ce que la philosophie ?, Paris, Les Editions de Minuit.

Dufour, D.R., 2003, "L’art de réduire les têtes » sur la nouvelle servitude de l'homme libéréà l'heure du capitalisme total, collections Méditations, Edition Denoël.

Dufrénois H., et Christian Miquel, 1996, La philosophie de l'exil, Paris, L'Harmattan.

Foucault, M., 1966, Les mots et les choses. Une archéologie des sciences humaines, Paris, Gallimard, nrf.

Foucault, M., 1975, Surveiller et punir. Naissance de la prison, Paris, Gallimard. Foucault, M., 1989, Résumé des cours (1970-1984), Paris, Juliart.

Fukuyama, F., 1992, La fin de l'histoire et le dernier homme, Paris, Flammarion. 
Habermas, J., 1978, L’espace public. «Archéologie de la publicité comme dimensionconstitutive de la société bourgeoise », Paris, Payot-Rivage.

Habermas, J., 2000, Après l'État-nation. Une nouvelle constellation politique, Paris, Fayard.

Hegel, G.W.F., 1940, Principe de la philosophie du droit, Paris, Gallimard.

Hardt, M. et Negri, A., 2000, Empire, Paris, Exils Editeurs.

Huntington, S., 1997, Le choc des civilisations, Paris, Editions Odile Jacob.

Jameson, F., 1991, Postmodernism, or, the cultural logic of late capitalism, Durham, Duke University Press.

Kant, E., 1945, Critique de la raison pure, Paris, P.U.F.

Lukacs, G., 1958, La destruction de la raison. Les débuts de l'irrationalisme moderne de Schelling à Nietzsche, Paris, L’Arche Editeur.

Lyotard, J.F., 1974, Economie libidinale, Paris, Les Editions de Minuit.

Lyotard, J.F., 1979, La condition postmoderne : rapport sur le savoir, Paris, Editions de Minuit.

Mengue, P., 2003, Deleuze et la question de la démocratie, Paris, L'Harmattan.

Negri, A., 2000, Kairos, Alma Venus et Multitude. Neuf leçons en formes d'exercices, Rome,Calmann - Lévy.

Ollier, F., 2001, « Capitalisme, barbarie esthétique et idéologie », X-Alta $n^{\circ} 4$, Social-sodomie.

Rorty, R., 1989, Contingence, ironie et solidarité, Paris, Armand Collin.

Vakakoulis, M., 2001, Le capitalisme postmoderne, Paris, coll. Marx confrontation, P.U.F.

Vattimo, G., 1987, La fin de la modernité. Nihilisme et herméneutique dans la culture postmoderne, Paris, Seuil. 